\title{
Synthesis of Polysubstituted Quinolines from the Acetates of Baylis-Hillman Adducts: Aza-Claisen Rearrangement as the Key Step
}

\author{
Seung Chan Kim, Saravanan Gowrisankar, and Jae Nyoung Kim* \\ Department of Chemistry and Institute of Basic Science, Chonnam National University, Gwangju 500-757, Korea \\ E-mail: kimjn@chonnam.ac.kr \\ Received April 16, 2005
}

Key Words : Quinolines, Baylis-Hillman adducts, Aza-Claisen rearrangement, PPA

Recently, we have reported a variety of chemical transformations of the Baylis-Hillman adducts into useful heterocyclic compounds. ${ }^{1,2}$ Very recently, we published the synthesis of 3-benzylquinolin-2-ols from the Baylis-Hillman adducts of ethyl acrylate bearing aniline moiety at the secondary position. ${ }^{1}$ 3-Benzylquinolin-2-ols could be formed via the sequential aza-Claisen rearrangement of the adduct in PPA, amide bond formation, and the following isomerization with the aid of DBU as shown in Scheme 1.

In this paper we would like to report our results on the synthesis of polysubstituted quinolines from the BaylisHillman adducts of alkyl vinyl ketones. As exemplified in Scheme 2, the required starting materials 2 were synthesized from the acetates of the Baylis-Hillman adducts of methyl vinyl ketone and ethyl vinyl ketone via the corresponding
DABCO salts according to the reported procedure. ${ }^{1 a, 3,4}$ In the reaction, however, cinnamyl amine derivatives $\mathbf{3}$ were isolated in variable yields (6-37\%) depending upon the nature of anilines (Table 1) as side products. ${ }^{5}$ The formation of primary adducts $\mathbf{3}$ from $\mathbf{2}$ under the reaction conditions can be explained by sequential addition and elimination of aniline, presumably due to the higher electrophilicity of the $\beta$-carbon (marked with asterisk) of $\mathbf{2}$ than the cases of ethyl acrylate (Scheme 1). ${ }^{5}$ As can be seen easily in the ratios of 2 and 3 (Table 1), the amounts of primary products $\mathbf{3}$ were gradually increased according to the increase of the nucleophilicity of anilines.

With the compound 2a in hand we examined the reaction in PPA at various reaction temperatures. Mixed solvent system of PPA and 1,2-dichloroethane was used in order to<smiles>CCOC(=O)C(=O)OC(C(=O)OCc1ccccc1)c1ccccc1</smiles><smiles>C=C(C(C)=O)C(OC(C)=O)c1ccccc1</smiles>

$1 \mathrm{a}$<smiles>C=C(C(=O)OCC)C([AlH2])c1ccccc1</smiles>

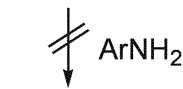<smiles>CCOC(=O)/C(=C/c1ccccc1)CNBr</smiles>

1. PPA

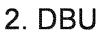<smiles>CCOC(=O)/C(=C/c1ccccc1)Cc1ccccc1N</smiles><smiles>C1CCCCC1</smiles><smiles>Oc1nc2ccccc2cc1Cc1ccccc1</smiles>

Scheme 1

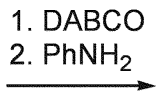<smiles>C=C(C(C)=O)C(Nc1ccccc1)c1ccccc1</smiles>

$2 a$<smiles>CC(=O)C(=Cc1ccccc1)CNc1ccccc1</smiles>

3a<smiles>CC(=O)/C(=C/c1ccccc1N)Cc1ccccc1</smiles>

1. PPA
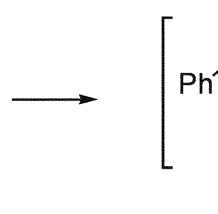<smiles>CC=C1Cc2ccccc2N=C1C</smiles><smiles>Cc1nc2ccccc2cc1Cc1ccccc1</smiles>

$6 a$

Scheme 2 
dissolve the starting materials efficiently (Experimental section). As expected we could obtain the dihydroquinoline compound 5a (42\%), which must be obtained via the sequential aza-Claisen rearrangement ${ }^{1,6}$ and condensation between the amino and the acetyl group. The optimum temperature was found to be around $70-80{ }^{\circ} \mathrm{C}$. But, the generated dihydroquinoline compound $\mathbf{5 a}$ was very unstable, which decomposed rapidly into intractable mixtures at room temperature.

Table 1. Synthesis of polysubstituted quinolines 6

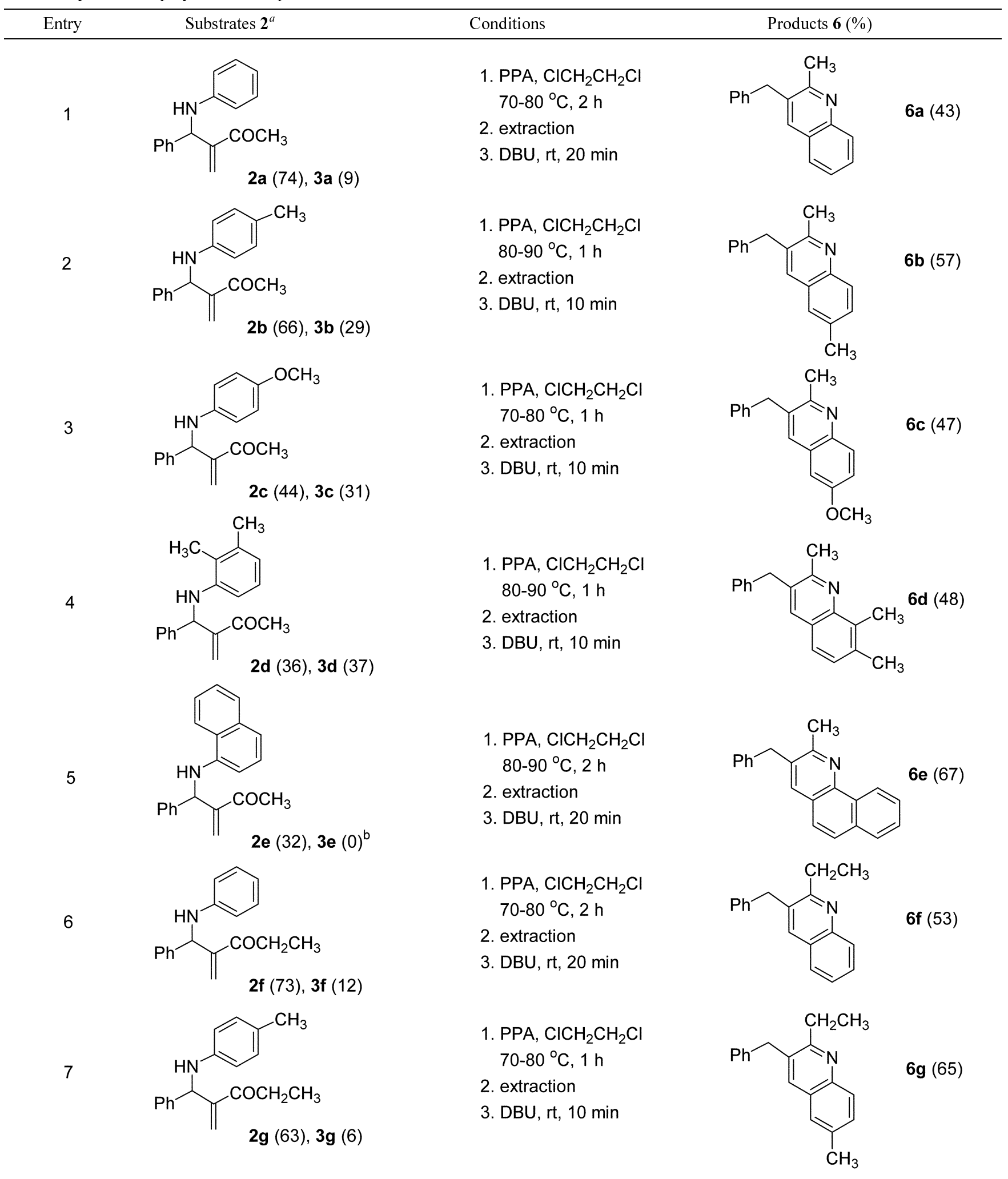


Fortunately, however, treatment of the dihydroquinoline intermediate 5a with DBU generated 2-methyl-3-benzylquinoline (6a) almost quantitatively. ${ }^{7}$ Thus, we carried out the synthesis of $\mathbf{6 a}$ without isolation of the dihydroquinoline intermediate 5a and we could obtain $\mathbf{6 a}$ in $43 \%$ yield. Similarly we prepared polysubstituted quinolines $\mathbf{6 b - g}$ and the results are summarized in Table 1.

As shown, quinolines 6b-g were obtained in $47-67 \%$ yields. In order to increase the yields of quinolines we examined other conditions including $p$ - $\mathrm{TsOH} / \mathrm{CH}_{3} \mathrm{CN} /$ reflux, $\mathrm{ZnCl}_{2} / \mathrm{xylene} /$ reflux, $\mathrm{LiClO}_{4} / 1$,2-dichloroethane/reflux. However, rearranged cinnamyl amine derivatives $\mathbf{3}$ were observed as the major products in all cases. The results showed that the aza-Claisen rearrangement of aniline moiety of 2 was facilitated in PPA in any way. However, we could not explain the reason at this stage.

In summary, we disclosed the synthesis of polysubstituted quinolines starting from Baylis-Hillman adducts of methyl vinyl ketone and ethyl vinyl ketone in moderate yields.

\section{Experimental Section}

Typical procedure for the synthesis of starting material 2a: To a stirred solution of the acetate of the Baylis-Hillman adduct $1 \mathrm{a}(436 \mathrm{mg}, 2 \mathrm{mmol})$ in aq THF $\left(5 \mathrm{~mL}, \mathrm{H}_{2} \mathrm{O} / \mathrm{THF}=\right.$ $1: 1)$ was added DABCO (246 $\mathrm{mg}, 2.2 \mathrm{mmol})$ at room temperature. After $30 \mathrm{~min}$, aniline $(280 \mathrm{mg}, 3 \mathrm{mmol})$ was added and stirred for $16 \mathrm{~h}$ at room temperature. After the usual workup and column chromatographic purification process (hexanes/ether, $10: 1$ ) we obtained 2a (372 mg, $74 \%$ ) and $3 \mathbf{a}(45 \mathrm{mg}, 9 \%)$, respectively. The other starting materials $\mathbf{2} \mathbf{b}-\mathbf{g}$ was prepared similarly and the spectroscopic data of $\mathbf{2 a - g}$ are as follows.

Compound 2a: $74 \%$; white solid, mp $95-97{ }^{\circ} \mathrm{C}$; IR ( $\mathrm{KBr}$ ) $3398,1674,1601,1500 \mathrm{~cm}^{-1} ;{ }^{1} \mathrm{H} \mathrm{NMR}\left(\mathrm{CDCl}_{3}, 300 \mathrm{MHz}\right) \delta$ $2.33(\mathrm{~s}, 3 \mathrm{H}), 4.11$ (br s, 1H), $5.51(\mathrm{~s}, 1 \mathrm{H}), 6.15(\mathrm{~s}, 1 \mathrm{H}), 6.23$ (s, 1H), 6.50-6.55 (m, 2H), 6.67-6.74 (m, 1H), 7.10-7.17 (m, 2H), 7.22-7.37 (m, 5H).

Compound 2b: $66 \%$; white solid, mp $111-112{ }^{\circ} \mathrm{C}$; IR (KBr) 3398, 1674, 1616, $1516 \mathrm{~cm}^{-1}$; ${ }^{1} \mathrm{H}$ NMR $\left(\mathrm{CDCl}_{3}, 300\right.$ MHz) $\delta 2.21(\mathrm{~s}, 3 \mathrm{H}), 2.32(\mathrm{~s}, 3 \mathrm{H}), 4.00(\mathrm{br} \mathrm{s}, 1 \mathrm{H}), 5.47(\mathrm{~s}$, $1 \mathrm{H}), 6.13(\mathrm{~s}, 1 \mathrm{H}), 6.21(\mathrm{~s}, 1 \mathrm{H}), 6.45(\mathrm{~d}, J=8.4 \mathrm{~Hz}, 2 \mathrm{H}), 6.94$ $(\mathrm{d}, J=8.4 \mathrm{~Hz}, 2 \mathrm{H}), 7.22-7.36(\mathrm{~m}, 5 \mathrm{H})$.

Compound 2c: $44 \%$; pale yellow solid, mp 78-79 ${ }^{\circ} \mathrm{C}$; IR (KBr) 3386, 1674, $1512 \mathrm{~cm}^{-1} ;{ }^{1} \mathrm{H}$ NMR $\left(\mathrm{CDCl}_{3}, 300 \mathrm{MHz}\right)$ $\delta 2.32(\mathrm{~s}, 3 \mathrm{H}), 3.72(\mathrm{~s}, 3 \mathrm{H}), 3.90(\mathrm{br} \mathrm{s}, 1 \mathrm{H}), 5.43(\mathrm{~s}, 1 \mathrm{H})$, $6.14(\mathrm{~s}, 1 \mathrm{H}), 6.22(\mathrm{~s}, 1 \mathrm{H}), 6.49(\mathrm{~d}, J=9.0 \mathrm{~Hz}, 2 \mathrm{H}), 6.74(\mathrm{~d}, J$ $=9.0 \mathrm{~Hz}, 2 \mathrm{H}), 7.23-7.37(\mathrm{~m}, 5 \mathrm{H}) ;{ }^{13} \mathrm{C} \mathrm{NMR}\left(\mathrm{CDCl}_{3}, 75\right.$ MHz) $\delta 26.67,55.68,58.45,114.59,114.71,126.09,127.39$, 127.55, 128.62, 140.93, 141.12, 148.47, 152.25, 199.19 .

Compound 2d: 36\%; pale yellow oil; IR (film) 3433, 1674, $1589 \mathrm{~cm}^{-1} ;{ }^{1} \mathrm{H}$ NMR $\left(\mathrm{CDCl}_{3}, 300 \mathrm{MHz}\right) \delta 2.05(\mathrm{~s}$, $3 \mathrm{H}), 2.27$ (s, 3H), 2.34 (s, 3H), 3.98 (br s, 1H), $5.54(\mathrm{~s}, 1 \mathrm{H})$, $6.13(\mathrm{~s}, 1 \mathrm{H}), 6.22(\mathrm{~s}, 1 \mathrm{H}), 6.31(\mathrm{~d}, J=8.1 \mathrm{~Hz}, 1 \mathrm{H}), 6.60(\mathrm{~d}, J$ $=7.5 \mathrm{~Hz}, 1 \mathrm{H}), 6.95(\mathrm{t}, J=7.8 \mathrm{~Hz}, 1 \mathrm{H}), 7.22-7.38(\mathrm{~m}, 5 \mathrm{H})$; ${ }^{13} \mathrm{C} \mathrm{NMR}\left(\mathrm{CDCl}_{3}, 75 \mathrm{MHz}\right) \delta 12.65,20.71,26.73,57.84$, $109.14,119.80,120.50,126.02$, 126.04, 127.45, 127.62,
$128.73,136.51,141.23,144.53,148.33,199.21$.

Compound 2e: $32 \%$; pale yellow oil; $\mathrm{IR}(\mathrm{KBr}) 3440,1670$ $\mathrm{cm}^{-1}$; ${ }^{1} \mathrm{H}$ NMR $\left(\mathrm{CDCl}_{3}, 300 \mathrm{MHz}\right) \delta 2.37(\mathrm{~s}, 3 \mathrm{H}), 4.87(\mathrm{~s}$, $1 \mathrm{H}), 5.71(\mathrm{~d}, J=3.6 \mathrm{~Hz}, 1 \mathrm{H}), 6.20$ (d, $J=1.2 \mathrm{~Hz}, 1 \mathrm{H}), 6.24$ $(\mathrm{s}, 1 \mathrm{H}), 6.43(\mathrm{dd}, J=6.6$ and $1.2 \mathrm{~Hz}, 1 \mathrm{H}), 7.22-7.47(\mathrm{~m}, 8 \mathrm{H})$, 7.77-7.83 (m, 2H); ${ }^{13} \mathrm{C} \mathrm{NMR}\left(\mathrm{CDCl}_{3}, 75 \mathrm{MHz}\right) \delta 26.74$, $57.95,105.90,117.92,119.88,123.45,124.81,125.72$, $126.08,126.42,127.55,127.81,128.70,128.82,134.23$, $140.85,141.58,147.78,199.28$.

Compound 2f: $73 \%$; white solid, mp $78-80{ }^{\circ} \mathrm{C}$; IR (KBr) $3398,1674,1601,1504 \mathrm{~cm}^{-1} ;{ }^{1} \mathrm{H} \mathrm{NMR}\left(\mathrm{CDCl}_{3}, 300 \mathrm{MHz}\right) \delta$ $1.03(\mathrm{t}, J=7.2 \mathrm{~Hz}, 3 \mathrm{H}), 2.56-2.84(\mathrm{~m}, 2 \mathrm{H}), 4.13$ (br s, $1 \mathrm{H})$, $5.51(\mathrm{~s}, 1 \mathrm{H}), 6.09(\mathrm{~s}, 1 \mathrm{H}), 6.20(\mathrm{~s}, 1 \mathrm{H}), 6.51-6.55(\mathrm{~m}, 1 \mathrm{H})$, 6.67-6.73 (m, 1H), 7.10-7.17 (m, 2H), 7.22-7.37 (m, 5H); ${ }^{13} \mathrm{C} \mathrm{NMR}\left(\mathrm{CDCl}_{3}, 75 \mathrm{MHz}\right) \delta 8.01,31.76,58.10,113.34$, $117.78,124.57,127.43,127.62,128.69,129.12,140.94$, 146.71, 147.68, 201.95 .

Compound 2g: 63\%; pale yellow oil; IR (KBr) 3398 , $1678,1616,1520 \mathrm{~cm}^{-1} ;{ }^{1} \mathrm{H}$ NMR $\left(\mathrm{CDCl}_{3}, 300 \mathrm{MHz}\right) \delta 1.02$ $(\mathrm{t}, J=7.2 \mathrm{~Hz}, 3 \mathrm{H}), 2.22(\mathrm{~s}, 3 \mathrm{H}), 2.56-2.84(\mathrm{~m}, 2 \mathrm{H}), 4.02(\mathrm{br}$ $\mathrm{s}, 1 \mathrm{H}), 5.47(\mathrm{~s}, 1 \mathrm{H}), 6.09(\mathrm{~s}, 1 \mathrm{H}), 6.20(\mathrm{~s}, 1 \mathrm{H}), 6.46(\mathrm{~d}, J=$ $8.4 \mathrm{~Hz}, 2 \mathrm{H}), 6.95(\mathrm{~d}, J=8.4 \mathrm{~Hz}, 2 \mathrm{H}), 7.24-7.37(\mathrm{~m}, 5 \mathrm{H}) ;{ }^{13} \mathrm{C}$ $\mathrm{NMR}\left(\mathrm{CDCl}_{3}, 75 \mathrm{MHz}\right) \delta 8.02,20.35,31.77,58.33,113.46$, $124.54,126.98,127.44,127.56$, 128.66, 129.62, 141.11, 144.46, 147.83, 202.02.

Typical procedure for the synthesis of polysubstituted quinoline 6a: A stirred solution of $\mathbf{2 a}(251 \mathrm{mg}, 1 \mathrm{mmol})$ in a mixed solvent of PPA $(1.0 \mathrm{~g})$ and 1,2-dichloroethane $(1 \mathrm{~mL})$ was heated to $70-80{ }^{\circ} \mathrm{C}$ for $2 \mathrm{~h}$. The reaction mixture was poured into cold aq $\mathrm{NaHCO}_{3}$ solution and extracted with ether. The organic layers were washed with water and dried with $\mathrm{MgSO}_{4}$. To the solution was added a catalytic amounts of DBU (15 mg, $0.1 \mathrm{mmol})$ and the solvent was removed under reduced pressure by rotary evaporator. During the solvent-removal process complete isomerization toward quinoline occurred. After column chromatographic purification process (hexanes/ether, $10: 1$ ) we obtained the desired 6a in $43 \%$ yield $(101 \mathrm{mg})$. The corresponding dihydroquinoline derivative $5 \mathbf{a}$ was isolated in a different reaction before treatment with DBU in $42 \%$ yield by rapid column purification process. The other quinolines $\mathbf{6 b}-\mathbf{g}$ was synthesized in a similar way and the spectroscopic data of 5a and 6a-g are as follows. Compound 5a: 42\%; pale yellow solid, mp 87$90{ }^{\circ} \mathrm{C}$; IR (KBr) 1597, 1562, $1493 \mathrm{~cm}^{-1}$; ${ }^{1} \mathrm{H} \mathrm{NMR}\left(\mathrm{CDCl}_{3}\right.$, $300 \mathrm{MHz}) \delta 2.51(\mathrm{~s}, 3 \mathrm{H}), 3.96(\mathrm{~d}, J=3.0 \mathrm{~Hz}, 2 \mathrm{H}), 7.04-7.15$ (m, 3H), 7.19-7.25 (m, 1H), 7.31-7.49 (m, 6H); ${ }^{13} \mathrm{C}$ NMR $\left(\mathrm{CDCl}_{3}, 75 \mathrm{MHz}\right) \delta 23.90,29.50,125.92,126.67,127.02$, $127.35,127.55,128.06,128.39$, 129.53, 131.29, 133.24, $135.87,142.83,164.78$.

Compound 6a: 43\%; pale yellow solid, mp $82-83{ }^{\circ} \mathrm{C}$; IR (KBr) 1601, 1493, $1415 \mathrm{~cm}^{-1}$; ${ }^{1} \mathrm{H}$ NMR $\left(\mathrm{CDCl}_{3}, 300 \mathrm{MHz}\right)$ $\delta 2.64(\mathrm{~s}, 3 \mathrm{H}), 4.13(\mathrm{~s}, 2 \mathrm{H}), 7.12-7.17(\mathrm{~m}, 2 \mathrm{H}), 7.20-7.34(\mathrm{~m}$, $3 \mathrm{H}), 7.41-7.47(\mathrm{~m}, 1 \mathrm{H}), 7.60-7.70(\mathrm{~m}, 2 \mathrm{H}), 7.75(\mathrm{~s}, 1 \mathrm{H})$, $8.01(\mathrm{~d}, J=8.4 \mathrm{~Hz}, 1 \mathrm{H}) ;{ }^{13} \mathrm{C} \mathrm{NMR}\left(\mathrm{CDCl}_{3}, 75 \mathrm{MHz}\right) \delta$ $23.78,39.37,125.97,126.68,127.28,127.45,128.52$, $128.86,129.00,129.06,132.93,136.06,139.08,146.87$, 159.07 . 
Compound 6b: $57 \%$; pale yellow solid, mp 58-60 ${ }^{\circ} \mathrm{C}$; IR (KBr) 2920, 1604, 1493, $1442 \mathrm{~cm}^{-1}$; ${ }^{1} \mathrm{H}$ NMR $\left(\mathrm{CDCl}_{3}, 300\right.$ $\mathrm{MHz}) \delta 2.48(\mathrm{~s}, 3 \mathrm{H}), 2.61(\mathrm{~s}, 3 \mathrm{H}), 4.11(\mathrm{~s}, 2 \mathrm{H}), 7.12-7.16$ (m, 2H), 7.19-7.33 (m, 3H), 7.43-7.47 (m, 2H), $7.66(\mathrm{~s}, 1 \mathrm{H})$, $7.90(\mathrm{~d}, J=9.0 \mathrm{~Hz}, 1 \mathrm{H}) ;{ }^{13} \mathrm{C} \mathrm{NMR}\left(\mathrm{CDCl}_{3}, 75 \mathrm{MHz}\right) \delta$ $21.45,23.42,39.13,125.95,126.39,127.22,127.96,128.58$, $128.81,130.98,132.60,135.26,135.44,138.97,145.21$, 157.78 .

Compound 6c: 47\%; white solid, mp $163-165{ }^{\circ} \mathrm{C}$; IR (KBr) 2920, 1604, 1493, $1227 \mathrm{~cm}^{-1}$; ${ }^{1} \mathrm{H} \mathrm{NMR}\left(\mathrm{CDCl}_{3}, 300\right.$ $\mathrm{MHz}) \delta 2.61(\mathrm{~s}, 3 \mathrm{H}), 3.87(\mathrm{~s}, 3 \mathrm{H}), 4.11(\mathrm{~s}, 2 \mathrm{H}), 6.96(\mathrm{~d}, J=$ $2.7 \mathrm{~Hz}, 1 \mathrm{H}), 7.14-7.35(\mathrm{~m}, 6 \mathrm{H}), 7.64(\mathrm{~s}, 1 \mathrm{H}), 7.90$ (d, $J=9.0$ $\mathrm{Hz}, 1 \mathrm{H}) ;{ }^{13} \mathrm{C} \mathrm{NMR}\left(\mathrm{CDCl}_{3}, 75 \mathrm{MHz}\right) \delta 23.22,39.13,55.44$, $104.80,121.25,126.42,128.08,128.63,128.91,129.72$, $132.99,134.83,138.98,142.67,156.09,157.24$.

Compound 6d: $48 \%$; pale yellow solid, mp $75-77{ }^{\circ} \mathrm{C}$; IR (KBr) 2920, 1616, 1493, 1450, $1427 \mathrm{~cm}^{-1}$; ${ }^{1} \mathrm{H} \mathrm{NMR}\left(\mathrm{CDCl}_{3}\right.$, $300 \mathrm{MHz}) \delta 2.46(\mathrm{~s}, 3 \mathrm{H}), 2.63(\mathrm{~s}, 3 \mathrm{H}), 2.75(\mathrm{~s}, 3 \mathrm{H}), 4.10(\mathrm{~s}$, $2 \mathrm{H}), 7.11-7.31(\mathrm{~m}, 6 \mathrm{H}), 7.44(\mathrm{~d}, J=8.1 \mathrm{~Hz}, 1 \mathrm{H}), 7.67$ (s, $1 \mathrm{H}) ;{ }^{13} \mathrm{C} \mathrm{NMR}\left(\mathrm{CDCl}_{3}, 75 \mathrm{MHz}\right) \delta 13.14,20.67,23.96$, $39.07,124.03,125.51,126.33,128.54,128.58,128.86$, $131.14,133.57,136.00,136.31,139.36,145.74,157.48$.

Compound 6e: $67 \%$; pale yellow solid, mp 99-101 ${ }^{\circ} \mathrm{C}$; IR (KBr) 1601, 1493, $1442 \mathrm{~cm}^{-1} ;{ }^{1} \mathrm{H}$ NMR $\left(\mathrm{CDCl}_{3}, 300 \mathrm{MHz}\right)$ $\delta 2.74(\mathrm{~s}, 3 \mathrm{H}), 4.18(\mathrm{~s}, 2 \mathrm{H}), 7.15-7.34(\mathrm{~m}, 5 \mathrm{H}), 7.56-7.74(\mathrm{~m}$, 4H), 7.78 (s, 1H), 7.85-7.88 (m, 1H), $9.30(\mathrm{~d}, J=8.1 \mathrm{~Hz}$, $1 \mathrm{H}) ;{ }^{13} \mathrm{C} \mathrm{NMR}\left(\mathrm{CDCl}_{3}, 75 \mathrm{MHz}\right) \delta 23.66,39.05,124.19$, $124.91,125.00,126.40,125.75,126.78,127.61,127.69$, $128.62,128.86,131.21,133.01,133.40,136.05,139.14$, 144.41, 157.23.

Compound 6f: $53 \%$; pale yellow solid, mp $62-64{ }^{\circ} \mathrm{C}$; IR (KBr) 2970, 1601, $1493 \mathrm{~cm}^{-1}$; ${ }^{1} \mathrm{H} \mathrm{NMR}\left(\mathrm{CDCl}_{3}, 300 \mathrm{MHz}\right)$ $\delta 1.31(\mathrm{t}, J=7.5 \mathrm{~Hz}, 3 \mathrm{H}), 2.95(\mathrm{q}, J=7.5 \mathrm{~Hz}, 2 \mathrm{H}), 4.18(\mathrm{~s}$, $2 \mathrm{H}), 7.13-7.18(\mathrm{~m}, 2 \mathrm{H}), 7.23-7.34(\mathrm{~m}, 3 \mathrm{H}), 7.41-7.47(\mathrm{~m}$, 1H), 7.60-7.70 (m, 2H), $7.76(\mathrm{~s}, 1 \mathrm{H}), 8.04(\mathrm{~d}, J=8.7 \mathrm{~Hz}$, $1 \mathrm{H}) ;{ }^{13} \mathrm{C} \mathrm{NMR}\left(\mathrm{CDCl}_{3}, 75 \mathrm{MHz}\right) \delta 13.12,29.10,38.58$, $125.70,126.42$, 127.04, 127.09, 128.52, 128.61, 128.67, $128.85,132.14,136.29,139.39,146.83,163.14$.

Compound 6g: 65\%; pale yellow oil; IR (film) 2970, 1604, $1493 \mathrm{~cm}^{-1}$; ${ }^{1} \mathrm{H} \mathrm{NMR}\left(\mathrm{CDCl}_{3}, 300 \mathrm{MHz}\right) \delta 1.29(\mathrm{t}, J=$ $7.5 \mathrm{~Hz}, 3 \mathrm{H}), 2.48(\mathrm{~s}, 3 \mathrm{H}), 2.93(\mathrm{q}, J=7.5 \mathrm{~Hz}, 2 \mathrm{H}), 4.15(\mathrm{~s}$, $2 \mathrm{H}), 7.12-7.16(\mathrm{~m}, 2 \mathrm{H}), 7.20-7.32(\mathrm{~m}, 3 \mathrm{H}), 7.44-7.48(\mathrm{~m}$, 2H), $7.66(\mathrm{~s}, 1 \mathrm{H}), 7.93(\mathrm{~d}, J=9.0 \mathrm{~Hz}, 1 \mathrm{H}) ;{ }^{13} \mathrm{C} \mathrm{NMR}$ $\left(\mathrm{CDCl}_{3}, 75 \mathrm{MHz}\right) \delta 13.16,21.46,28.99,38.55,125.92$, $126.36,127.09,128.18,128.57,128.83,130.90,132.04$, $135.39,135.73,139.50,145.39,162.12$.
Acknowledgments. This work was supported by the grant (R-05-2003-000-10042-0) from the Basic Research Program of the Korea Science and Engineering Foundation (Now controlled under the authority of Korea Research Foundation). Spectroscopic data was obtained from the Korea Basic Science Institute, Gwangju branch.

\section{References and Notes}

1. For our recent papers on the reaction of Baylis-Hillman adducts bearing aniline moiety, see (a) Lee, C. G.; Lee, K. Y.; Lee, S.; Kim, J. N. Tetrahedron 2005, 61, 1493. (b) Lee, C. G.; Lee, K. Y.; Gowrisankar, S.; Kim, J. N. Tetrahedron Lett. 2004, 45,7409 .

2. For our recent publications on the synthesis of quinoline derivatives, see (a) Lee, K. Y.; Kim, J. M.; Kim, J. N. Tetrahedron 2003, 59, 385. (b) Kim, J. N.; Chung, Y. M.; Im, Y. J. Tetrahedron Lett. 2002, 43, 6209. (c) Kim, J. N.; Kim, H. S.; Gong, J. H.; Chung, Y. M. Tetrahedron Lett. 2001, 42, 8341. (d) Kim, J. N.; Lee, H. J.; Lee, K. Y.; Kim, H. S. Tetrahedron Lett. 2001, 42, 3737. (e) Kim, J. N.; Lee, K. Y.; Kim, H. S.; Kim, T. Y. Org. Lett. 2000, 2, 343. (f) Gowrisankar, S.; Na, J. E.; Lee, M. J.; Kim, J. N. Bull. Korean Chem. Soc. 2005, 26, 319. (g) Lee, K. Y.; Kim, J. M.; Kim, J. N. Bull. Korean Chem. Soc. 2002, 23, 1493. (h) Lee, K. Y.; Kim, J. N. Bull. Korean Chem. Soc. 2002, 23, 939. (i) Na, J. E.; Lee, K. Y.; Park, D. Y.; Kim, J. N. Bull. Korean Chem. Soc. 2005, 26,323 and further references for the synthesis of polysubstituted quinolines cited therein.

3. For the introduction of nucleophiles at the secondary position of the Baylis-Hillman adducts by using the corresponding DABCO salt, see (a) Kim, J. M.; Lee, K. Y.; Kim, J. N. Bull. Korean Chem. Soc. 2004, 25, 328. (b) Lee, K. Y.; Kim, T. H.; Kim, J. N. Bull. Korean Chem. Soc. 2004, 25, 1966 and further references cited therein.

4. For the synthesis of Baylis-Hillman adducts bearing the aniline moiety, see (a) Rajesh, S.; Banerji, B.; Iqbal, J. J. Org. Chem. 2002, 67, 7852. (b) Bauchat, P.; Le Rouille, E.; Foucaud, A. Bull. Chim. Soc. Fr. 1991, 267. (c) Buchholz, R.; Hoffmann, H. M. R. Helv. Chim. Acta 1991, 74, 1213.

5. For the acetates of Baylis-Hillman adducts of ethyl acrylate (Scheme 1), we could not observe the formation of cinnamyl amine derivatives during the preparation of the corresponding secondary adducts.

6. (a) Castro, A. M. M. Chem. Rev. 2004, 104, 2939 and further references cited therein. (b) Jolidon, S.; Hansen, H.-J. Helv. Chim. Acta 1977, 60, 978. (c) Anderson, W. K.; Lai, G. Synthesis 1995, 1287.

7. (a) Cho, C. S.; Kim, B. T.; Kim, T.-J.; Shim, S. C. Chem. Commun. 2001, 2576. (b) Cho, C. S.; Kim, B. T.; Choi, H.-J.; Kim, T.-J.; Shim, S. C. Tetrahedron 2003, 59, 7997. (c) Palimkar, S. S.; Siddiqui, S. A.; Daniel, T.; Lahoti, R. J.; Srinivasan, K. V. J. Org. Chem. 2003, 68, 9371. (d) Abbiati, G.; Beccalli, E. M.; Broggini, G.; Zoni, C. Tetrahedron 2003, 59, 9887. 